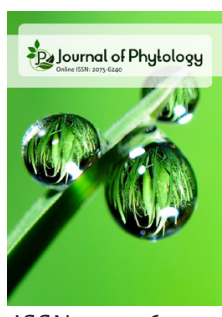

ISSN: $2075-6240$

\title{
Virtual screening of anticancer efficacy of phloretin against apoptotic targets - An In Silico molecular docking study
}

\author{
Thangavelu Ranjanamala', Krishanan Vanmathiselvi'*, \\ Ramanathan Shreemaya ${ }^{2}$, Sundarajan Nagarajan Rajeswari³, \\ Casimeer C Sangeetha ${ }^{4}$, Alaa Yousef Ghidan', Fatima Yousel Ghidan ${ }^{6}$
}

Received: August 01, 2021 Revised: October 20, 2021 Accepted: October 25, 2021 Published: November 19, 2021

*Corresponding Author: Krishanan Vanmathiselvi E-mail:kvmsel@gmail.com
'PG and Research Department of Microbiology, Sri Akilandeswari Womens Arts and Science College, Wandiwash-604408, Tamil Nadu, India, ${ }^{2}$ Department of Biotechnology, DY Patil Deemed to be University, Navi Mumbai-400614, India, '3Department of Botany, Seethalakshmiachi College for Women, Pallathur, Karaikudi, Tamilnadu 630107, India, “Department of Physics, Sri Padmavati Mahila Visvavidyalayam (Women's University), Tirupati,Andhra Pradesh 517502, India, ${ }^{5}$ Nanotechnology and Microbiology, Research and Development Center, The Higher Council for Science and Technology, Amman, 11941, Jordan, ${ }^{6} \mathrm{Head}$ of Quality and Medication Saftey Department, King Abdullah Medical City, Al Mashair, Makkah 24246, Saudi Arabia

\begin{abstract}
Recent advances demonstrate phytochemicals to be a potent anticancer therapeutic agent as various anti-cancer targets. This study depicts the anti-cancer potential against certain crucial common cancer targets leading to cancer cell proliferation and survival. The main objective of this study is to study the anti-cancer potential of phloretin against certain cancer targets. Ligand analysis was performed and Phloretin was chosen as the experimental ligand and Bcl-2, NF Kappa B, Carbonic anhydrase I (CA-1), Inducible Nitric Oxide Synthase (iNOS), Endothelial Nitric oxide synthase (eNOS), Caspase 3, and Caspase 9 proteins were chosen as targets. Induced fit molecular docking was performed by the use of Glide 6.5 software (Schrodinger-2015). The docked poses were further evaluated based on binding energy, Conformational changes, and the amino acid residues involved in the protein-ligand interaction. The docking results depicted that phloretin showed notable binding affinity especially with carbonic anhydrase I, ENOS, and INOS. It also showcased significant potential against Caspase 3 and NF Kappa, thereby showing its potential as an effective anticancer therapeutics. During this study, the Inhibitory potential of Phloretin was studied as a result of this molecular docking study. This Insilico study revealed the binding efficiency of phloretin against the aforementioned targets. In vitro analysis is required for further validation of this data.
\end{abstract}

KEYWORDS: Computer-aided drug discovery; Phloretin; Molecular docking, Anti-cancer activity.

\section{INTRODUCTION}

Worldwide, cancer is the primary cause of death and it presents itself as a hindrance and leads to a significant decrease in life expectancy. Female breast cancer is the most frequently diagnosed cancer, followed by lung, colorectal, prostate, and stomach cancer (Sung et al., 2021). Regardless of recent advancements in the development of modern medicine, Cancer is still the root cause of mortality worldwide. Presently, the major treatment options for cancer are chemotherapy, radiotherapy, and surgery (Pratheeshkumar et al., 2015). There has been a constant development of novel anti-cancer drugs. However, the use of synthetic drugs hasn't ameliorated the treatment efficiency and there has been no overall improvement in the survival rate of the patients (Choudhari et al., 2019). The major disadvantages of chemically synthesized drugs are high toxicity, drug resistance, and fatal effect on the non-targeted tissues. Due to this, there is a need for the development of novel therapeutics for a safer and effective treatment approach.

Initially, traditional Drug designing approaches took around 7 to 10 years via trial and error methods for screening of potential compounds. This methodology was labor-intensive and timeconsuming. After, finding potential compounds, It took around 10 years to perform clinical trials to develop a novel drug for a particular disease. For this purpose, a Computer-aided drug

Copyright: $\odot$ The authors. This article is open access and licensed under the terms of the Creative Commons Attribution License (http://creativecommons.org/licenses/by/4.0/) which permits unrestricted, use, distribution and reproduction in any medium, or format for any purpose, even commercially provided the work is properly cited. Attribution - You must give appropriate credit, provide a link to the license, and indicate if changes were made. 
designing method was developed using Molecular mechanics to understand the composition of the ligands. Multiple ligands can be chosen and around 200 - 500 compounds can be screened simultaneously using virtual screening. Molecular docking can be performed in silico, wherein the interaction between small molecules and proteins is analyzed at an atomic level. It is used in the characterization of the binding site of target proteins as well as to understand the inner workings of human diseases at the molecular level (Mariadoss et al., 2018). Compounds with higher efficacy can be chosen by performing Blind docking. Potential compounds can be further tested during clinical trials. Nowadays, Target - Specific inhibitors can be designed by targeting specific domains which correspond to certain functions implicated by that protein.

In this study, various targets are analyzed for understanding its potential as a biomarker/anti-cancer target for developing a novel drug for various types of cancers. The BCL-2 family comprises pro-apoptotic factors (BAX, BAK, BIM, BID, and BAD), which induce apoptosis, and anti-apoptotic factors (BCL-2, BCL-xL), which inhibits apoptosis. BCL-2 is an attractive target to investigate and develop novel anti-cancer therapeutic for various types of cancers. BCL-2 protein is an antiapoptotic protein that impairs apoptosis, thereby promoting cancer progression. It consists of 4 domains $\mathrm{BH}-4$. The $\mathrm{BHl}$, $\mathrm{BH} 2$, and $\mathrm{BH} 3$ domains interact to configure the hydrophobic groove and it accommodates the $\mathrm{BH} 3$ of pro-apoptotic proteins like BAX, thereby inhibiting apoptosis. BCL-2 protein is generally overexpressed in various cancers, thereby inhibiting the release of cytochrome $\mathrm{C}$ into the cytosol and leads to subsequent caspase activation (Giam et al., 2008).

$\mathrm{NF \kappa B}$ (nuclear factor kappa beta) is an inducible transcription factor and it is known to escalate cell proliferation, angiogenesis, and metastasis thereby making the cell resistant to anticancer drugs and chemotherapy treatment in breast cancer, colon cancer, and lymphoma (Xia et al., 2018). BRCAl silencing in breast cancer triggers phosphorylation of the Ser 536 site of p65 and processing of $\mathrm{pl} 00 / \mathrm{p} 52$, resulting in constitutive activation of the canonical $\mathrm{NF} \kappa \mathrm{B}$ pathway (p65/p50) and noncanonical NFкB pathway (p100/p52), along with nuclear translocation and accumulation of p52/RelB, which can enhance MCF1 cell proliferation (Sau et al., 2016). It leads to suppression of apoptosis via inhibition of apoptotic machinery in normal and cancerous cells (Baud $\&$ Karin, 2009). Activation of NFKB is shown to influence inflammation-associated cancers. Through binding of specific dimers of activated $\mathrm{NF} \kappa \mathrm{B}$ to promoters of pro-inflammatory genes, activation of canonical and noncanonical $\mathrm{NF} \kappa \mathrm{B}$ pathways accelerates tumour development and enhances the synthesis of proinflammatory proteins (Xia et al., 2018).

Carbonic anhydrases are known to mediate the $\mathrm{pH}$ in tumor cells by modulating the proton and bicarbonate concentrations, thereby inducing cellular survival and proliferation (Mboge et al., 2018). It is highly expressed in lung cancer. Lung cancer is difficult to detect and diagnose at early stages. CT scans are employed for the detection of lung cancer in high-risk patients, but it lacks sensitivity and specificity and it leads in false-positive results.
Carbonic anhydrases-1 was significantly upregulated and differentially expressed in lung cancer samples (Wang et al., 2016). Thus, Carbonic anhydrases - 1 demonstrates to be a potent biomarker for the early diagnosis of lung cancer.

Caspases are proteases and play a crucial aspect in inducing apoptosis. There are two major pathways involved regarding the activation of caspases-intrinsic and extrinsic pathways. The intrinsic pathway is characterized by the release of cytochrome $\mathrm{C}$ and interacts with adapter protein Apaf-1 (Apoptotic protease activating factor- 1 ) and forms apoptosome complex and induces activation of caspase 9 and ultimately activating caspase 3 protein (Parrish et al., 2013). Downregulation of caspase $3 /$ caspase 9 is generally associated with overexpression of the anti-apoptotic protein like BCL-2, which inhibits mitochondria from releasing cytochrome $\mathrm{c}$ in the cytosol and thus prevents the caspase activation, thereby inhibiting apoptosis and induces drug resistance in cancer cells. High Caspase-3 expression was significantly related to the PR and HER-2 status of patients (Yang et al., 2018). It is associated with prognosis for patients with breast cancer (Yang et al., 2018). Studies show that overexpression of caspase 9 induces its activation and leads to aberrant apoptosis in pituitary cells and HeLa cells (Druskovic et al., 2006). So, a specific inhibitor of caspase 9 is required to control its expression in vitro.

iNOS (Inducible nitric oxide synthase) is an enzyme that is proficient in NO (nitric oxide) production in response to various stimuli especially in response to certain endotoxins and endogenous pro-inflammatory cytokines (Lechner et al., 2005). Upregulated iNOS expression is mediated via NF $\kappa \mathrm{B}$ and many transcription factors. The elevated expression of iNOS was significantly associated with angiogenesis, chemotherapeutic resistance, metastasis, and immune resistance in some malignant tumors (Liao et al., 2019), e.g., targeting angiogenesis pathway in colorectal cancer (Gao et al., 2019), breast cancer (Fahey \& Girotti, 2017; Garrido et al., 2017), pancreatic cancer (Wang et al., 2016), gastric cancer (Chen et al., 2006), and melanoma (Lopez-Rivera et al., 2014). It is a promising biomarker for anti-cancer therapy. Endothelial nitric oxide synthase (eNOS) is proficient in the endothelial synthesis of nitric oxide (NO) and has been observed to depict inflammation, angiogenesis, apoptosis, and metastasis. Increased expression of eNOS and iNOSis was observed in malignant tissues of breast cancer (Loibl et al., 2002). eNOS induces lymphatic metastasis in many human cancers (Lahdenranta et al., 2009).

Phytochemicals, natural compounds obtained from plants depict great potential as anti-cancer agents. They depict low toxicity, target specific mechanism of action, and bioavailability of the drug and can be utilised to treat a variety of cancers. Potent phytochemicals inhibit cancer-related signalling pathways, but they also work in well with chemotherapy and radiotherapy (Pratheeshkumar et al., 2015). Phytochemicals work by modulating molecular pathways that have been linked to cancer growth and progression. Increased antioxidant status, carcinogen inactivation, inhibition of proliferation, induction of cell cycle arrest and apoptosis; and immune system control are 
some of the specific mechnanisam for phytochemical mediated cancer treatment method (Choudhari et al., 2019).

Phloretin (2',4',6'-Trihydroxy-3-(4-hydroxyphenyl)propiophenone), a natural polyphenolic compound, is found in apples, pears, and the Rosaceae family species (Lu \& Foo, 1997; Mariadoss et al., 2019a). Phloretin has demonstrated various pharmacological effects, especially anti-oxidant, anti-inflammatory (Huang et al., 2015), and antitumor properties (Mariadoss et al., 2019b). Phloretin depicts anti-cancer activity in vitro in human esophageal cancer cells via mitochondria-dependent pathway by inducing downregulation of BCL-2 and upregulation of $\mathrm{p} 53$ protein thereby promoting apoptosis in cancer cells (Duan et al., 2017). It also induces apoptosis in human liver cancer cells (Wu et al., 2009). It is observed that phloretin inhibits cell growth and induces apoptosis by increases mitochondrial permeability thereby activating caspase 9 leading to caspase 3 activation (Park et al., 2007). Phloretin proves to be a potent anti-cancer therapeutic agent (Mariadoss et al., 2019c). During this research study, phloretin is chosen as an experimental ligand and it is docked with BCL-2, NFкB, carbonic anhydrase 1 (CA-1), iNOS, eNOS, caspase 3, and caspase 9 which are crucial anti-cancer targets to assess the binding efficiency of phloretin and analyze its anti-cancer efficacy against these targets.

\section{MATERIALS AND METHODS}

\section{Protein Preparation}

3D structure of BAX, Bcl-2, NF Kappa B, Carbonic anhydrase I, Inducible Nitric Oxide Synthase, Endothelial Nitric oxide synthase, Caspase 3, and Caspase 9 were retrieved from the Protein Data Bank (PDB). It is a repository with the collection of protein 3D structures generated by NMR spectroscopy and X-ray crystallography techniques. Protein preparation was conducted by deletion of water molecules and addition of missing hydrogen bonds and correct bond orders were assigned to non-standard residues. Following this, the Optimization of hydroxyl group and amine groups of the amino acids and optimization of H-bonds on the target proteins were carried out. OPLS3e force field was used for the energy minimization process. The non-hydrogen atoms were minimized until the average root mean square deviation reached the default value of $0.3 \AA$.

\section{Active Site Prediction}

The active sites of the proteins were predicted using the Uniprot database, Interpro scan server, and the Sitemap module of Schrodinger. Uniprot is a crucial database to perceive and analyze diverse information of target proteins. Interpro scan is a sequence analysis application server that provides information on the functional domain, active sites, and putative sites. The sitemap module of Schrodinger is another source to predict the binding sites of the protein.vIn this research study, the active site residues of Carbonic anhydrase 1, INOX, ENOX, Caspase 3 , and Caspase 9 were predicted by Uniprot and Interpro scan web server. Whereas the active site residues of $\mathrm{BCl}-2$ and $\mathrm{NF}$ Kappa B were predicted by Sitemap.

\section{Ligand Preparation}

The 3D structure of compound Phloretin (PubChem ID: 4788) was downloaded from the PubChem database. The physical-chemical properties of the compound were analyzed using the Swiss-ADME server. The LigPrep program provides a versatile and robust procedure for the preparation of the ligands (Repasky et al., 2007). The optimization of the ligand was achieved by deploying OPLS3e as the force field.

\section{Domain Analysis}

Domains are capable to fold their structure alone and the domain is the functional part of the protein. From the concept of structural biology, the domains are the conserved region of the protein. The protein domain analysis was carried out using the Pfam database to predict the functional domain region of the selected protein.

\section{Receptor Grid Generation}

The position and size of the active site are represented by the formation of receptor grids. For this, a receptor grid generation module was used for the docking process where compounds have to be docked using a centroid of selecting residues of the proteins. It is a crucial step to perform before undertaking molecular docking.

\section{Induced Fit docking using XP Glide Algorithm}

Induced Fit docking is incorporated in this research study via the use of the XP(Extra Precision) scoring algorithm. The ligand docking module was incorporated for the docking of compound Phloretin with the target proteins using the XP GLIDE module of Schrödinger. XP Glide involves an extensive sampling of poses and a scoring function based on a rigorous assessment of proteinligand interactions (Repasky et al., 2007). To comprehensively sample ligand degrees of freedom, an anchor-and-grow technique has been used to refine the predicted docking modes. Anchor fragments of the docked ligand, typically ring or other rigid fragments, are chosen from the set of poses output from an initial docking with SP Glide designed to obtain a large diversity of docked structures. Ligand postures are regrown one side chain at a time from these anchor positions at very high resolutions. Combining high-scoring individual conformations at each side chain yields a list of potential compounds. The candidate molecules' energy is minimized, and ligand poses are rated using the Emodel pose-selection function (Repasky et al., 2007). The default scaling factors for the van der Waal's radii in Glide (1.0 for receptor and 0.8 for ligands) have been selected to strike a balance between accurate docking/scoring and compensation for the lack of receptor flexibility (Repasky et al., 2007). The top-scoring structures are subjected to grid-based water scoring methodology, and the entire XP GlideScore scoring function and identification of structural motifs are computed. 


\section{RESULTS AND DISCUSSION}

\section{Protein preparation and Active Site Prediction}

The targets were chosen based on molecular function and biological processes of the proteins in cancer progression. 3D structures of all seven proteins were downloaded in PDB format from PDB (Protein Bank Database) for further analysis were shown in Figure 1.

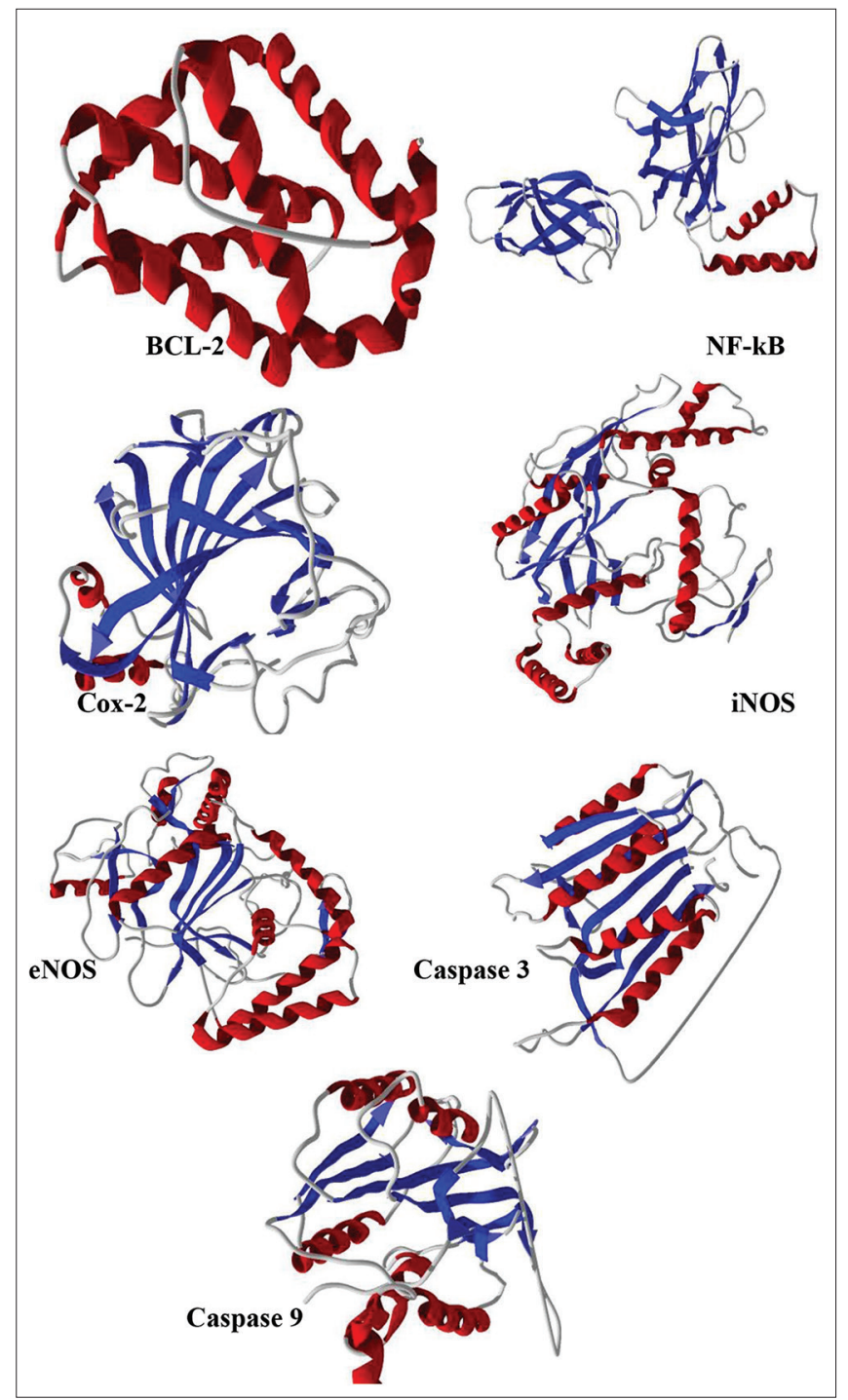

Figure 1: 3D structures of target proteins

\section{Domain Analysis}

The functional domain analysis of the target proteins was performed using the Pfam database and this information is shown in Table 1. These details were crucial in determining the active sites and their amino acid residues in each of our target proteins.

\section{Active site Prediction of Selected Proteins}

The amino acid residues of the active sites of target proteins were predicted using Uniprot, Interproscan, and Sitemap. The active site of Carbonic anhydrase 1, INOX, ENOX, Caspase 3 , and Caspase 9 were predicted by Uniprot and Interproscan. And remaining proteins $\mathrm{BAX}, \mathrm{BCl}-2$, and NF Kappa B binding sites were predicted by Sitemap (Table 2).

\section{Ligand Preparation}

The ligand molecule was downloaded from the PubChem database (Figure 2).

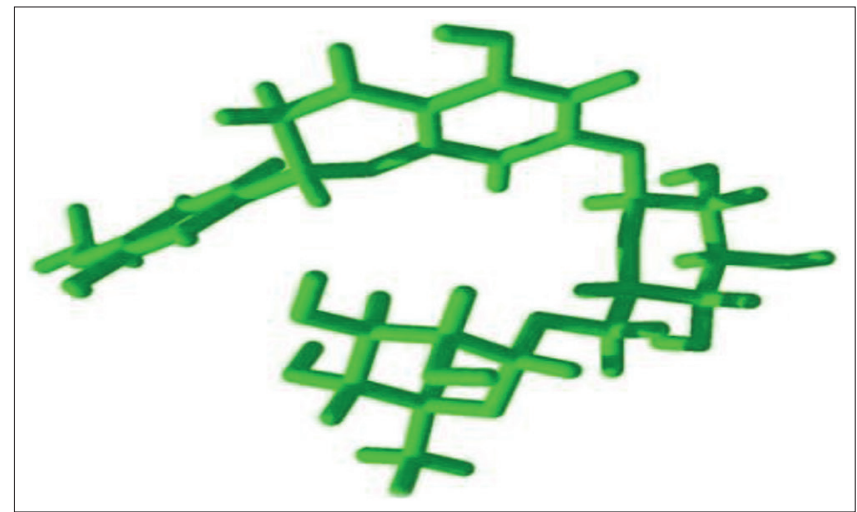

Figure 2: 3D structure of phloretin

Table 1: Domain analysis for the given anti-cancer targets

\begin{tabular}{lllc}
\hline Sr.No & Protein Name & Domain Name & Domain Region \\
\hline 1. & Bcl-2 protein (Q07817) & Bcl-2 & $90-188$ \\
2. & NF Kappa B (Q00653) & RHD_DNA_bind & $40-220$ \\
3. & Human carbonic anhydrase & Carb_anhydrase & $12-261$ \\
& I (P00915) & & \\
4. & INOS (P35228) & NO_synthase & $82-508$ \\
5. & ENOS (P29474) & NO_synthase & $119-481$ \\
6. & Caspase 3 (P42574) & Peptidase_C14 & $43-167$ \\
7. & Caspase 9 (P55211) & Peptidase_C14 & $160-412$ \\
\hline
\end{tabular}

Table 2: Prediction of the active site and its amino acid residues

\begin{tabular}{lllr}
\hline Sr.No & Protein Name & PDB ID & Active site/Binding site residues \\
\hline 1. & Bcl-2 protein (Q07817) & 4IEH & $55,58,59,62,63,66,67,103,104,105,107,108,157,161-163$. \\
2. & NF Kappa B (Q00653) & IA3Q & $94,95,103-105,107-109,117-119,153,154,156,157,160,193$ \\
3. & Human carbonic & SGMM & $62,64,65,67,91,92,94,96,119,121,122,131,135,141,143,198-200$, \\
& anhydrase-1 (P00915) & & 207,209 \\
4. & INOS (P35228) & INOS & $118,200,347,370,372,381,382,463,491$ \\
5. & ENOS (P29474) & INM & $184,247,353,354,356,357,368,366,447,475$ \\
6. & Caspase-3 (P42574) & IJXQ & $64,121,122,161,163,168,204-209,213,214$ \\
7. & Caspase-9 (P55211) & & $180,237,238,285,287,292,352-357,361,362$ \\
\hline
\end{tabular}




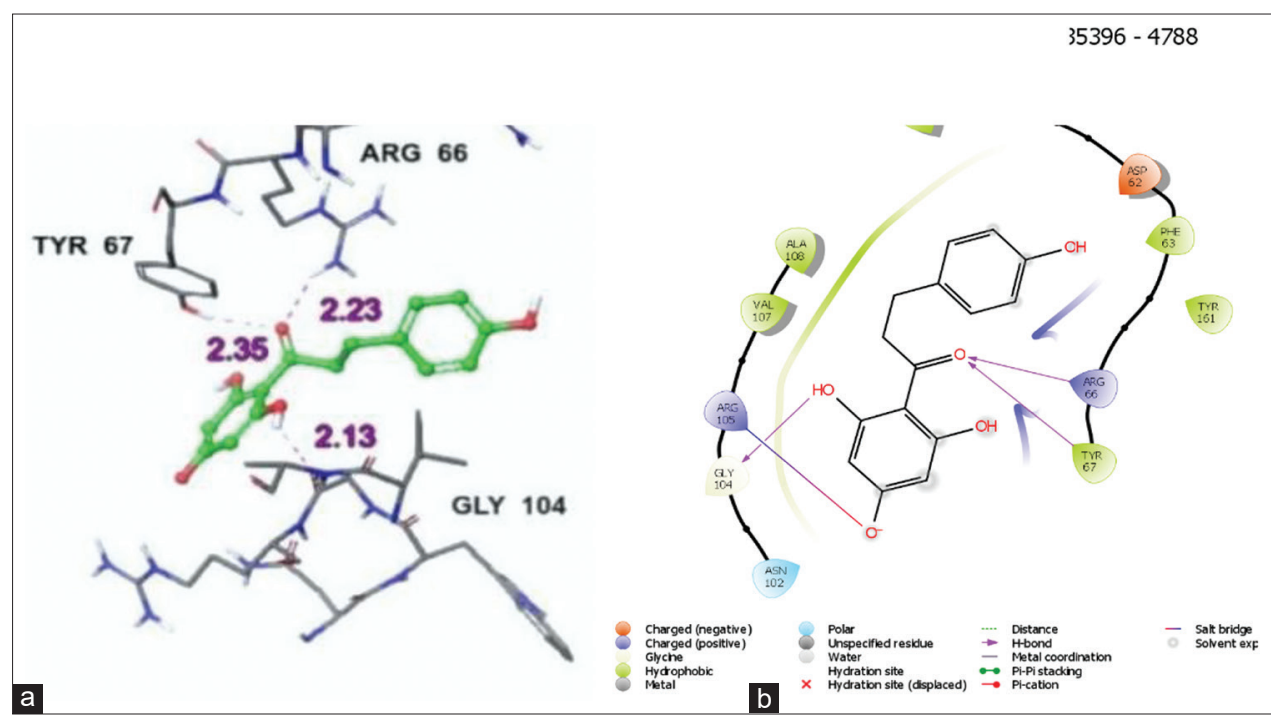

Figure 3: Interaction of Phloretin with BCL-2 protein (4IEH) (a) Visualization of Interaction of phloretin with BCL-2 protein. (b) Visualization of various non-covalent molecular interactions

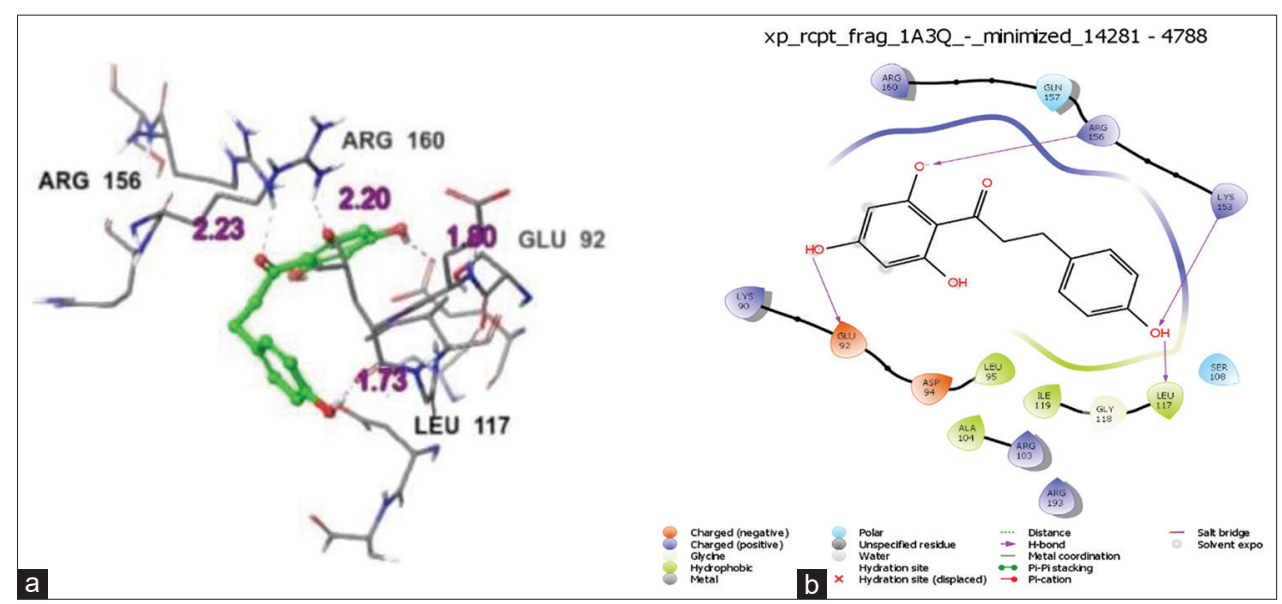

Figure 4: Interaction of Phloretin with NFKB protein (1A3Q). (a) Visualization of Interaction of phloretin with NFKB protein. (b) Visualization of various non-covalent molecular interactions

Table 3: Prediction of ADMETox properties of Phloretin

\begin{tabular}{lc}
\hline Properties & Specifications \\
\hline Ligand Name & Phloretin \\
Molecular Weight & 274.27 \\
LogP0/w & 2.63 \\
H bond Donor & 4 \\
H bond Acceptor & 5 \\
Polar surface Area & 97.99 \\
R05 & Yes \\
Log S & -3.38 \\
GI Absorption & High \\
Lead likeness & Yes \\
\hline
\end{tabular}

\section{Prediction of ADMETox properties}

The ADMETox properties were predicted using the SwissADME server. The predicted values (Table 3) suggest phloretin as an appropriate compound to be considered for docking based on its physical-chemical properties, oral bioavailability, and lead likeness.

\section{Molecular Docking}

In this research study, crucial anti-cancer targets like B cell lymphoma-2 (BCL-2), Nuclear factor kappa beta (NFKB), carbonic anhydrase 1(CA-1), iNOS (inducible nitric oxide synthase), eNOS (endothelial nitric oxide synthase), caspase-3 and Caspase-9 was docked against phloretin to analyze its anti-cancer potential. The induced-fit molecular docking process was carried out using the XP (Extra precision) method by the GLIDE module of Schrödinger. Phloretin depicts good binding affinity against all anti-cancer targets. Various interactions of Phloretin like h-bond, pi-pi with various proteins, bond lengths of interactions, and dock score values of dock score and interacting residues are shown in Table 4. The 


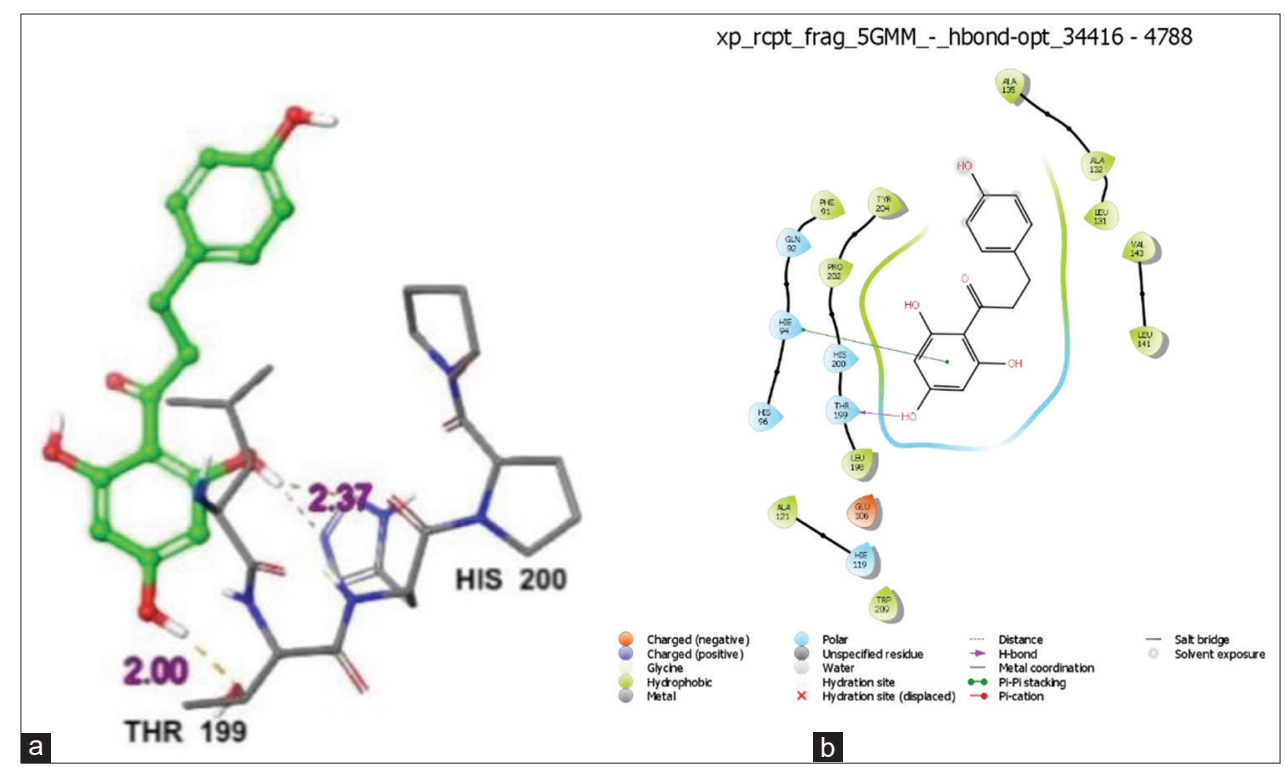

Figure 5: Interaction of Phloretin with Carbonic anhydrase 1 protein (a) Visualization of Interaction of phloretin with carbonic anhydrase 1 protein. (b)Visualization of various non-covalent molecular interactions

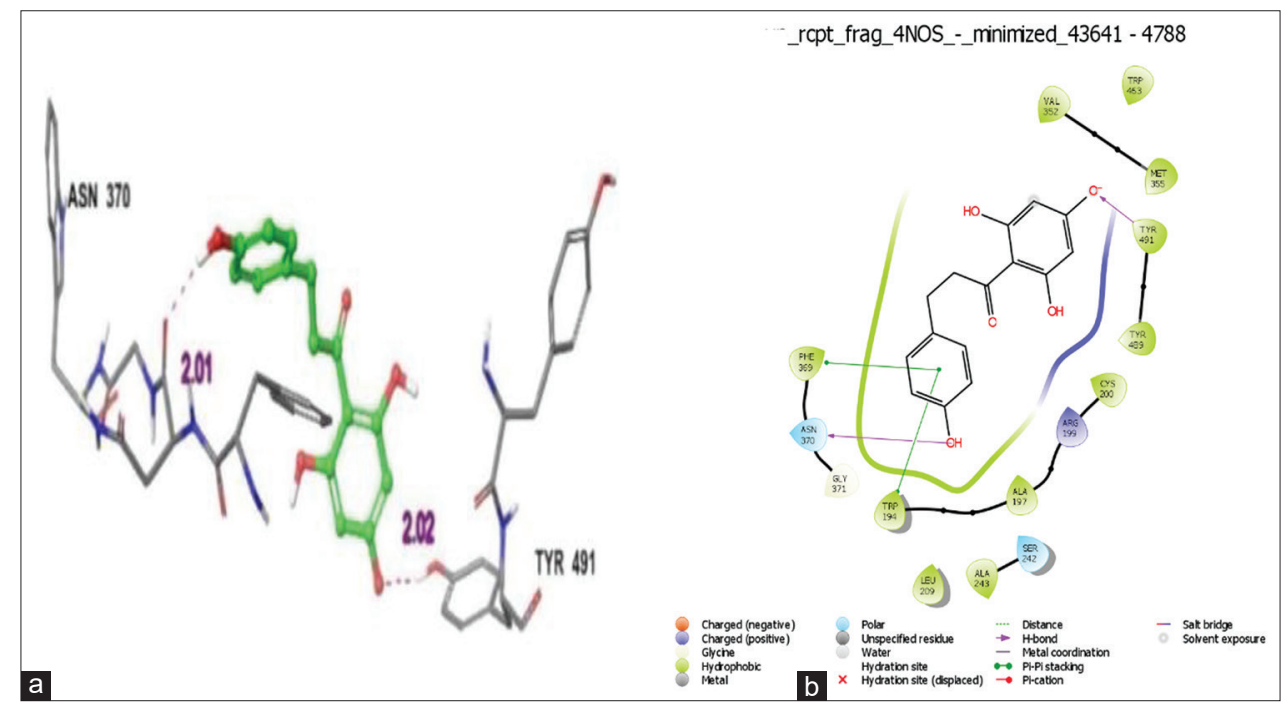

Figure 6: Interaction of Phloretin with Inducible nitric oxide synthase protein (a) Visualization of Interaction of phloretin with iNOS protein. (b) Visualization of various non-covalent molecular interactions

Table 4: Phloretin docked with selected proteins associated with cancer

\begin{tabular}{|c|c|c|c|}
\hline $\begin{array}{l}\text { Protein } \\
\text { Name }\end{array}$ & $\begin{array}{l}\text { Dock Score } \\
\text { (Kcal/mol) }\end{array}$ & Interacting Residues & Bond length $(\AA)$ \\
\hline $\mathrm{Bcl}-2$ protein & -4.3 & Arg 66, Tyr 67, Gly 104 & $2.23,2.35,2.13$ \\
\hline NF Kappa B & -5.3 & $\begin{array}{l}\text { Glu 92, Leu 117, Lys } \\
\text { 153, Arg } 156\end{array}$ & $\begin{array}{l}1.80,1.73 \\
1.86,2.20\end{array}$ \\
\hline $\begin{array}{l}\text { Carbonic } \\
\text { anhydrase } 1\end{array}$ & -6.2 & His $94(\mathrm{Pi}-\mathrm{Pi})$, Thr 199 & $4.93,2.00$ \\
\hline INOS & -7.2 & $\begin{array}{l}\text { Trp 194, Phe 369, Asn } \\
\text { 370, Tyr } 491\end{array}$ & $\begin{array}{l}3.46,3.98 \\
2.01,2.02\end{array}$ \\
\hline ENOS & -7.3 & $\begin{array}{l}\text { Trp } 178 \text { (2), Ser 354, } \\
\text { Tyr } 475 \text { (2) }\end{array}$ & $\begin{array}{l}3.46,4.25 \\
1.96,2.03\end{array}$ \\
\hline Caspase 3 & -5.4 & $\begin{array}{l}\text { Trp } 206 \text { (pi-pi), Arg } \\
207, \text { Asn 208, Asp 211, } \\
\text { Trp 214, Phe } 250\end{array}$ & $\begin{array}{l}5.30,2.03 \\
2.71,1.59 \\
1.81,2.09\end{array}$ \\
\hline Caspase 9 & -3.0 & $\begin{array}{l}\text { Trp 348, Glu 351, Asp } \\
\text { 355, Lys } 380\end{array}$ & $\begin{array}{l}2.26,1.66 \\
1.72,2.18\end{array}$ \\
\hline
\end{tabular}

J Phytol • 2021 • Vol 13 docking analysis showed that phloretin interacted with BCL-2 protein through a carbonyl group, wherein $\mathrm{C}=\mathrm{O}$ interacted with amino acid residue ARG 66 of hydrogen bond interactions and the hydroxyl group of ligand interacted with GLY 104 are similar to the residues observed on the interaction of inhibitor N-heteroaryl sulfonamide against BCL-2 (Toure et al., 2013). Phloretin also interacted with another binding site residue Tyr 67 with an overall docking score of $4.3 \mathrm{Kcal} / \mathrm{mol}$ (Figure 3). The docking analysis showed that phloretin interacted with $\mathrm{NF} \kappa \mathrm{B}$ protein through the hydroxyl group interacted with amino acid residues LEU 117, LYS 153, and GLY 92, which depicted hydrogen bond interaction. The overall docking score is $-5.3 \mathrm{Kcal} / \mathrm{mol}$ (Figure 4 ).

The docking analysis showed that phloretin interacted with Carbonic anhydrase I, wherein the hydroxyl group interacted 


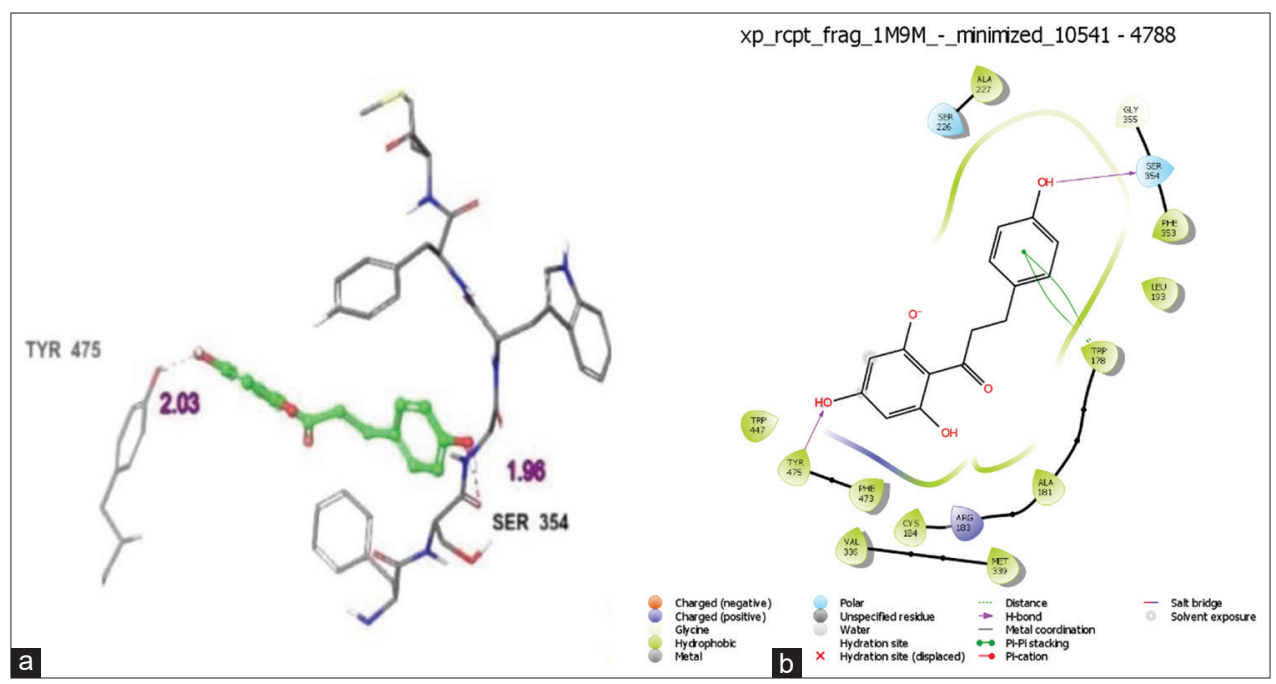

Figure 7: Interaction of Phloretin with Endothelial nitric oxide synthase protein (a) Visualization of Interaction of phloretin with eNOS protein. (b) Visualization of various non-covalent molecular interactions

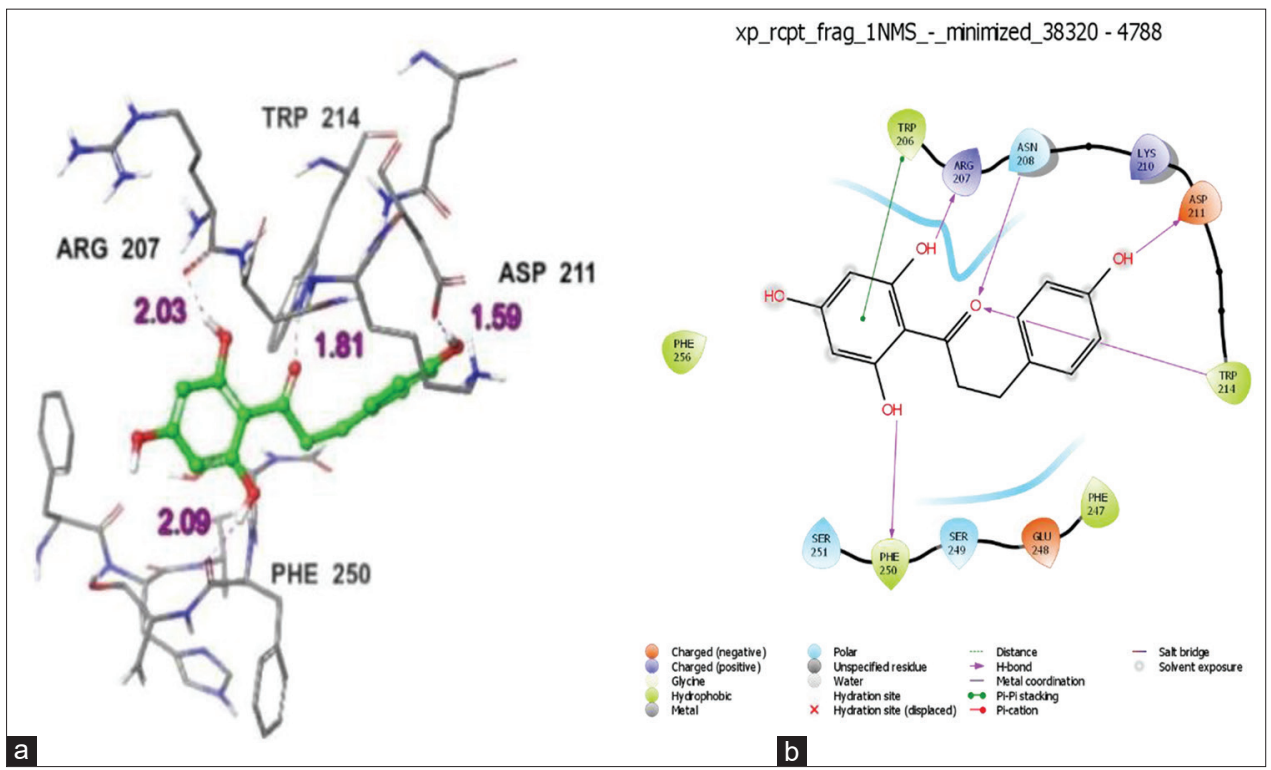

Figure 8: Interaction of Phloretin with Caspase 3 protein. (a) Visualization of Interaction of phloretin with caspase 3 protein. (b) Visualization of various non-covalent molecular interactions

with amino acid residues THR 199 which depicted hydrogen bond interaction and benzene ring of phloretin interacts with HIE 94 (Histidine with hydrogen on the epsilon nitrogen) which depicted pi-pi interaction. The crucial interactions are similarly depicted by binding inhibitor Polmacoxib against carbonic anhydrase 1 (Kim et al., 2016). It resulted in an overall docking score of $-6.2 \mathrm{Kcal} / \mathrm{mol}$ (Figure 5).

The docking analysis showed that phloretin interacted with Inducible nitric oxide synthase (iNOS), wherein the carbon-oxygen bond interacted with TYR 491 and the hydroxyl group interacted with amino acid residues ASN 370, the benzene ring of phloretin interacts with TRP 194, PHE 369 and depicted pi-pi interaction. It resulted in an overall docking score of $-7.2 \mathrm{Kcal} / \mathrm{mol}$ (Figure 6).
The docking analysis showed that phloretin interacted with the endothelial nitric oxide synthase (eNOS), wherein hydroxyl group interacted with amino acid residues TYR 475 and SER 354 which depicted hydrogen bond interaction, and benzene ring of phloretin interacted with TRP 178 which depicted pi-pi interaction. It resulted in an overall docking score of $-7.3 \mathrm{Kcal} /$ mol (Figure 7).

The docking analysis showed that phloretin with Caspase 3 protein, wherein hydroxyl group interacted with amino acid residues ARG 207, ASP 211, and PHE 250 and carbonyl group $\mathrm{C}=\mathrm{O}$ interacted with ASN 208 and TRP 214, which depicted hydrogen bond interaction. It resulted in an overall docking score of $-5.4 \mathrm{Kcal} / \mathrm{mol}$ (Figure. 8). 


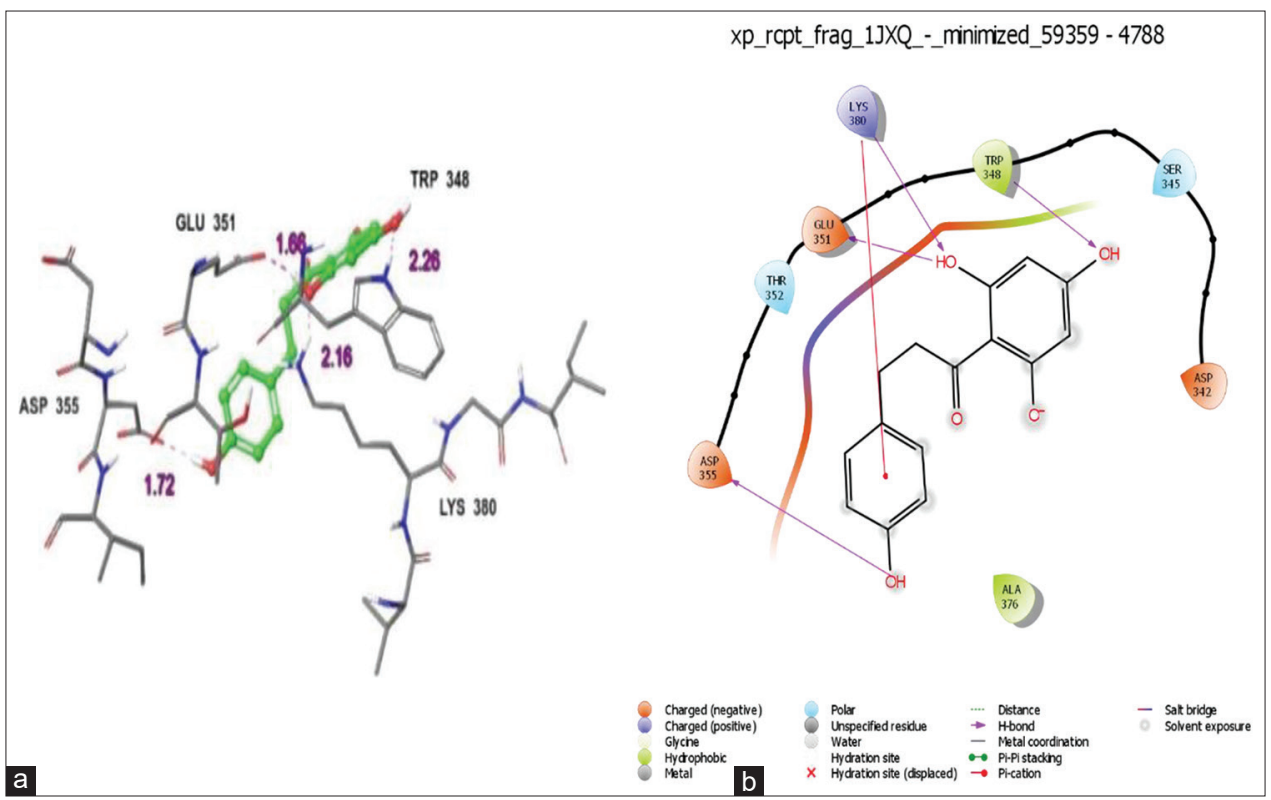

Figure 9: Interaction of Phloretin with Caspase 9 protein (a) Visualization of Interaction of phloretin with caspase 3 protein. (b) Visualization of various non-covalent molecular interactions

The docking analysis showed that phloretin with Caspase 9 protein, wherein hydroxyl group interacted with amino acid residues TRP 348, LYS 380, GLU 351, and ASP 355, which depicted hydrogen bond interaction. It resulted in an overall docking score of $-3.0 \mathrm{Kcal} / \mathrm{mol}$ (Figure 9). Overall, Phloretin showed good binding affinity and showed good interaction with the active site residues with the respective target proteins.

\section{CONCLUSION}

The results from the present research study suggest that phloretin proves to be a potent anti-cancer therapeutic and depicts significant binding efficacy and affinity against these crucial anti-cancer targets. The anti-cancer mechanism of phloretin against these targets cannot be fully understood by an in silico study. Further analysis via in-vitro techniques are required to understand the anti-cancer potential of phloretin. Phloretin showed good binding values and can be considered and further developed as a potent anti cancer therapeutic to aid in the treatment of various cancers.

\section{AUTHOR'S CONTRIBUTION}

All authors contributed equality in carrying out the research study and the development of this paper.

\section{ACKNOWLEDGEMENTS}

The authors are thankful to the authorities of Sri Akilandeswari womens arts and science college, Wandiwash-604408, Tamilnadu, India for providing necessary support to carry out this research work.

\section{REFERENCES}

Baud, V., \& Karin, M. (2009). Is NF-kappaB a good target for cancer therapy? Hopes and pitfalls. Nat Rev Drug Discov, 8(1), 33-40. https://doi. org/10.1038/nrd2781

Chen, C. N., Hsieh, F. J., Cheng, Y. M., Chang, K. J., \& Lee, P. H. (2006). Expression of inducible nitric oxide synthase and cyclooxygenase $\square 2$ in angiogenesis and clinical outcome of human gastric cancer. Journal of Surgical Oncology, 94(3), 226-233. https://doi.org/10.1002/ jso.20372

Choudhari, A. S., Mandave, P. C., Deshpande, M., Ranjekar, P., \& Prakash, O. (2019). Phytochemicals in Cancer Treatment: From Preclinical Studies to Clinical Practice. Front Pharmacol, 10, 1614. https://doi. org/10.3389/fphar.2019.01614

Druskovic, M., Suput, D., \& Milisav, I. (2006). Overexpression of caspase-9 triggers its activation and apoptosis in vitro. Croatian Medical Journal, 47(6), 832-840.

Duan, H., Wang, R., Yan, X., Liu, H., Zhang, Y., Mu, D., Han, J., \& Li, X. (2017). Phloretin induces apoptosis of human esophageal cancer via a mitochondria-dependent pathway. Oncology Letters, 14(6), 6763-6768. https://doi.org/10.3892/ol.2017.7037

Fahey, J. M., \& Girotti, A. W. (2017). Nitric oxide-mediated resistance to photodynamic therapy in a human breast tumor xenograft model: Improved outcome with $\mathrm{NOS}_{2}$ inhibitors. Nitric Oxide, 62, 52-61. https://doi.org/10.1016/..niox.2016.12.003

Gao, Y., Zhou, S., Xu, Y., Sheng, S., Qian, S. Y., \& Huo, X. (2019). Nitric oxide synthase inhibitors $1400 \mathrm{~W}$ and L-NIO inhibit angiogenesis pathway of colorectal cancer. Nitric Oxide, 83, 33-39. https://doi.org/10.1016/i. niox.2018.12.008

Garrido, P., Shalaby, A., Walsh, E. M., Keane, N., Webber, M. Keane, M. M., Sullivan, F. J., Kerin, M. J., Callagy, G., Ryan, A. E., \& Glynn, S. A. (2017). Impact of inducible nitric oxide synthase (iNOS) expression on triple negative breast cancer outcome and activation of EGFR and ERK signaling pathways. Oncotarget, 8(46), 80568-80588. https://doi.org/10.18632/oncotarget.19631

Giam, M., Huang, D. C., \& Bouillet, P. (2008). BH3-only proteins and their roles in programmed cell death. Oncogene, 27, S128-136. https:// doi.org/10.1038/onc.2009.50

Huang, W. C. Dai, Y. W., Peng, H. L., Kang, C. W., Kuo, C. Y. \& Liou, C. J. (2015). Phloretin ameliorates chemokines and ICAM-1 expression via blocking of the NF-KB pathway in the TNF- $\alpha$-induced $\mathrm{HaCaT}$ human keratinocytes. International Immunopharmacology, 27(1), 32-37. https://doi.org/10.1016/j.intimp.2015.04.024 
Kim, H. T., Cha, H., \& Hwang, K. Y. (2016). Structural insight into the inhibition of carbonic anhydrase by the COX-2-selective inhibitor polmacoxib (CG100649). Biochemical and Biophysical Research Communications 478(1), 1-6. https://doi.org/10.1016/j.bbrc.2016.07.114

Lahdenranta, J., Hagendoorn, J., Padera, T. P., Hoshida, T., Nelson, G., Kashiwagi, S., Jain, R. K., \& Fukumura, D. (2009). Endothelial nitric oxide synthase mediates lymphangiogenesis and Iymphatic metastasis. Cancer Research, 69(7), 2801-2808. https://doi. org/10.1158/0008-5472.CAN-08-4051

Lechner, M., Lirk, P., \& Rieder, J. (2005). Inducible nitric oxide synthase (iNOS) in tumor biology: the two sides of the same coin. Seminars in Cancer Biology, 15(4), 277-289. https://doi.org/10.1016/j. semcancer.2005.04.004

Liao, W., Ye, T., \& Liu, H. (2019). Prognostic value of inducible nitric oxide synthase (iNOS) in human cancer: A systematic review and metaanalysis. BioMed Research International, 2019, 6304851. https://doi. org/10.1155/2019/6304851

Loibl, S., von Minckwitz, G., Weber, S., Sinn, H. P., Schini-Kerth, V. B., Lobysheva, I., Nepveu, F., Wolf, G., Strebhardt, K., \& Kaufmann, M. (2002). Expression of endothelial and inducible nitric oxide synthase in benign and malignant lesions of the breast and measurement of nitric oxide using electron paramagnetic resonance spectroscopy. Cancer, 95(6), 1191-1198. https://doi.org/10.1002/cncr.10817

Lopez-Rivera, E., Jayaraman, P., Parikh, F., Davies, M. A., Ekmekcioglu, S., Izadmehr, S., Milton, D. R., Chipuk, J. E., Grimm, E. A., Estrada, Y., Aguirre-Ghiso, J., \& Sikora, A. G. (2014). Inducible nitric oxide synthase drives mTOR pathway activation and proliferation of human melanoma by reversible nitrosylation of TSC2. Cancer Research, 74(4), 1067-1078. https://doi.org/10.1158/0008-5472. CAN-13-0588

Lu, Y., \& Foo, L. Y. (1997). Identification and quantification of major polyphenols in apple pomace. 59(2), 187-194. https://doi.org/10.1016/ s0308-8146(96)00287-7

Mariadoss, A. V. A., Dhanabalan, A. K., Munusamy, H., Gunasekaran, K., \& David, E. (2018). In Silico Studies towards Enhancing the Anticancer Activity of Phytochemical Phloretin Against Cancer Drug Targets. Current Drug Therapy, 13(2), 174-188. https://doi.org/10.2174/1574 885513666180402134054

Mariadoss, A. V. A., Vinayagam, R., Xu, B., Venkatachalam, K., Sankaran, V., Vijayakumar, S., David, E. (2019b). Phloretin loaded chitosan nanoparticles enhance the antioxidants and apoptotic mechanisms in DMBA induced experimental carcinogenesis. Chemico-Biological Interactions, 308, 11-19. https://doi.org/10.1016/j.cbi.2019.05.008

Mariadoss, A. V. A., Vinyagam, R., Rajamanickam, V., Sankaran, V., Venkatesan, S., \& David, E. (2019c). Pharmacological aspects and potential use of phloretin: A systemic review. Mini Reviews in Medicinal Chemistry, 19(13), 1060-1067. https://doi.org/10.2174/13 89557519666190311154425

Mariadoss, A., Vinayagam, R., Senthilkumar, V., Paulpandi, M., Murugan, K., Xu, B., K M, G., Kotakadi, V. S., \& David, E. (2019a). Phloretin loaded chitosan nanoparticles augments the $\mathrm{pH}$-dependent mitochondrial-mediated intrinsic apoptosis in human oral cancer cells. International Journal of Biological Macromolecules, 130, 9971008. https://doi.org/10.1016/j.ijbiomac.2019.03.031

Mboge, M. Y., Mahon, B. P., McKenna, R., \& Frost, S. C. (2018). Carbonic Anhydrases: Role in pH Control and Cancer. Metabolites, 8(1). https:// doi.org/10.3390/metabo8010019
Park, S. Y., Kim, E. J., Shin, H. K., Kwon, D. Y., Kim, M. S., Surh, Y. J., \& Park, J. H. (2007). Induction of apoptosis in HT-29 colon cancer cells by phloretin. Journal of Medicinal Food, 10(4), 581-586. https://doi. org/10.1089/jmf.2007.116

Parrish, A. B., Freel, C. D., \& Kornbluth, S. (2013). Cellular mechanisms controlling caspase activation and function. Cold Spring Harbor Perspectives in Biology, 5(6). https://doi.org/10.1101/cshperspect. a008672

Pratheeshkumar, P., Son, Y. O., Korangath, P., Manu, K. A., \& Siveen, K. S. (2015). Phytochemicals in Cancer Prevention and Therapy. BioMed Research International, 2015, 324021. https://doi. org/10.1155/2015/324021

Repasky, M. P., Shelley, M., \& Friesner, R. A. (2007). Flexible Ligand Docking with Glide. Current Protoco/s in Bioinformatics, 18(1), 8.12.11 18.12.36. https://doi.org/https://doi.org/10.1002/0471250953. bi0812s18

Sau, A., Lau, R., Cabrita, M. A., Nolan, E., Crooks, P. A., Visvader, J. E. \& Pratt, M. A. (2016). Persistent Activation of NF-kappaB in BRCA1Deficient Mammary Progenitors Drives Aberrant Proliferation and Accumulation of DNA Damage. Ce// Stem Cell, 19(1), 52-65. https:// doi.org/10.1016/j.stem.2016.05.003

Sung, H., Ferlay, J., Siegel, R. L., Laversanne, M., Soerjomataram, I., Jemal, A., \& Bray, F. (2021). Global Cancer Statistics 2020: GLOBOCAN Estimates of Incidence and Mortality Worldwide for 36 Cancers in 185 Countries. CA: A Cancer Journal for Clinicians, 71(3), 209-249. https://doi.org/10.3322/caac.21660

Touré, B. B., Miller-Moslin, K., Yusuff, N., Perez, L., Doré, M., Joud, C. Michael, W., DiPietro, L., van der Plas, S., McEwan, M., Lenoir, F., Hoe, M., Karki, R., Springer, C., Sullivan, J., Levine, K., Fiorilla, C., Xie, X., Kulathila, R., Herlihy, K., Porter, D, \& Visser, M. (2013). The role of the acidity of N-heteroaryl sulfonamides as inhibitors of bcl-2 family protein-protein interactions. ACS Medicinal Chemistry Letters, 4(2), 186-190. https://doi.org/10.1021/ml300321d

Wang, D. B., Lu, X. K., Zhang, X., Li, Z. G., \& Li, C. X. (2016). Carbonic anhydrase 1 is a promising biomarker for early detection of nonsmall cell lung cancer. Tumour Biology, 37(1), 553-559. https://doi. org/10.1007/s13277-015-3834-z

Wang, J., He, P., Gaida, M., Yang, S., Schetter, A. J., Gaedcke, J., Ghadimi, B. M., Ried, T., Yfantis, H., Lee, D., Weiss, J. M., Stauffer, J., Hanna, N., Alexander, H. R., \& Hussain, S. P. (2016). Inducible nitric oxide synthase enhances disease aggressiveness in pancreatic cancer. Oncotarget, 7(33), 52993. https://doi.org/10.18632/ oncotarget.10323

Wu, C. H., Ho, Y. S., Tsai, C. Y., Wang, Y. J., Tseng, H., Wei, P. L., Lee, C. H., Liu, R. S., \& Lin, S. Y. (2009). In vitro and in vivo study of phloretininduced apoptosis in human liver cancer cells involving inhibition of type II glucose transporter. International Journal of Cancer, 124(9), 2210-2219. https://doi.org/10.1002/ijc.24189

Xia, L., Tan, S., Zhou, Y., Lin, J., Wang, H., Oyang, L., Tian, Y., Liu, L., Su, M., Wang, H., Cao, D., \& Liao, Q. (2018). Role of the NFKB-signaling pathway in cancer. OncoTargets and Therapy, 11, 2063-2073. https:// doi.org/10.2147/OTT.S161109

Yang, X., Zhong, D. N., Qin, H., Wu, P. R., Wei, K. L., Chen, G., He, R. Q. \& Zhong, J. C. (2017). Caspase-3 over-expression is associated with poor overall survival and clinicopathological parameters in breast cancer: a meta-analysis of 3091 cases. Oncotarget, 9(9), 8629-8641. https://doi.org/10.18632/oncotarget.23667 